\title{
COMPROMETIMENTO ÓSSEO DO JOELHO PÓS-TRAUMA: AVALIAÇÃO PELA RESSONÂNCIA MAGNÉTICA*
}

\author{
Carla Martins De Grossi ${ }^{1}$, Edson Marchiori², Alair Augusto S.M.D. dos Santos ${ }^{3}$
}

Resumo Neste trabalho são apresentados 50 casos de trauma do joelho submetidos a ressonância magnética, no período de janeiro de 1996 a dezembro de 1997. Foram avaliados o aspecto e a incidência das principais alterações ósseas, correlacionando-as com os mecanismos de agressão e com os dados clínicos, e demonstrando as principais lesões associadas. As contusões ósseas foram os achados mais comuns, sendo encontradas em 38 indivíduos (76\%). As fraturas osteocondrais ocorreram em cinco pacientes (10\%). As fraturas ósseas foram detectadas em cinco casos $(10 \%)$, sendo três deles associados a contusão de outros compartimentos ósseos adjacentes. A condromalácia da patela mostrou-se presente em apenas dois indivíduos (4\%). Concluímos que a ressonância magnética é o método de escolha no estudo por imagem para o diagnóstico das lesões ósseas no trauma do joelho.

Unitermos: Ressonância magnética. Lesões ósseas. Trauma. Joelho.

Abstract Bone injuries in the post-traumatic knee: evaluation by magnetic resonance imaging.

This paper presents the results of 50 patients with trauma of the knee submitted to magnetic resonance imaging in the period of January 1996 to December 1997. The appearance and incidence of the main bone lesions were evaluated and correlated with the mechanisms of aggression and with clinical data. Associated lesions were also evaluated. Bone contusions were the most commonly findings encountered and were observed in 38 patients $(76 \%)$. Osteochondral fractures occurred in five patients $(10 \%)$ and bone fractures were detected in five patients $(10 \%)$, of which two were associated with contusions in other adjoining bone compartments. Chondromalacia of the patella was observed only in two patients $(4 \%)$. We concluded that magnetic resonance imaging is the imaging method of choice for the diagnosis of bone lesions in patients with trauma of the knee.

Key words: Magnetic resonance imaging. Bone injuries. Trauma. Knee.

\section{INTRODUÇÃO}

Atualmente, a ressonância magnética (RM) desempenha importante papel na avaliação do trauma do joelho, pela sua capacidade de obtenção de cortes finos e multiplanares, com diferentes ponderações de imagem, sendo capaz de identificar lesões meniscais, ligamentares, tendinosas e ósseas ${ }^{\mathbf{1}, \mathbf{2})}$. Mostra-se também vantajosa por não utilizar radiação ionizante, não ser um método invasivo e não haver necessidade de preparo prévio ou

* Trabalho realizado no Serviço de Radiologia do Hospital Santa Cruz (Santa Cruz Scan)/Beneficência Portuguesa de Niterói, RJ, e no Serviço de Radiodiagnóstico do Hospital Universitário Clementino Fraga Filho (HUCFF) da Universidade Federal do Rio de Janeiro (UFRJ), Rio de Janeiro, RJ.

1. Mestre em Radiologia pela UFRJ.

2. Professor Titular de Radiologia da Universidade Federal Fluminense (UFF), Coordenador Adjunto do Curso de Pós-graduação em Radiologia da UFRJ.

3. Professor e Chefe do Serviço de Radiologia da UFF Médico do Serviço de Radiologia do Hospital Santa Cruz (Santa Cruz Scan)

Endereço para correspondência: Prof. Dr. Edson Marchiori. Rua Thomaz Cameron, 438, Valparaíso. Petrópolis, RJ, 25685-120. E-mail: edmarchiori@zipmail.com.br

Aceito para publicação em 13/2/2001. da administração de meio de contraste iodado ou paramagnético, evitando-se possíveis reações a esses contrastes ${ }^{(3)}$.

Lesões ósseas ocultas à radiografia convencional podem ser demonstradas pela RM, sendo incluídas neste grupo as contusões ósseas e as fraturas osteocondrais. Estas lesões são responsáveis por quadros álgicos e têm valor prognóstico importante, necessitando ser reconhecidas e tratadas para se evitar sequielas graves. Estas áreas, indetectáveis pela radiografia convencional, representam sítios de hemorragia, edema ou hiperemia óssea. Lesões meniscais ${ }^{(4-6)}$, ligamentares, tendinosas e derrames intra-articulares estão usualmente associadas a essas injúrias ósseas, sendo facilmente identificadas pela $\mathrm{RM}^{(7)}$.

Neste trabalho, foram estudados pela $\mathrm{RM}$ os principais tipos de lesões ósseas do joelho causadas por trauma, bem como avaliadas as demais lesões associadas, a partir de estudo retrospectivo de 50 pacientes, realizado no período de janeiro de 1996 a dezembro de 1997.

\section{PACIENTES, MATERIAL E MÉTODOS}

Foram estudados 50 pacientes encaminhados ao Santa Cruz Scan (Hospital Santa Cruz/Beneficência Portuguesa de Niterói, RJ), no período de janeiro de 1996 a dezembro de 1997, com história de trauma no joelho.

$\mathrm{O}$ aparelho de RM utilizado na avaliação destes pacientes foi o Vectra, da General Electric, de 0,5 Tesla (T).

Foram realizadas sequiências multiplanares nos planos axial, coronal e sagital com cortes de $4 \mathrm{~mm}$ de espessura, com campo de interesse ("field of view" FOV) de 14 a $16 \mathrm{~cm}$, número de excitações (NEX) de 1 ou 2, "flip angle" de 10 a 12 graus e matriz em torno de $256 \times$ 128 a $256 \times 192$.

As seqüências multiplanares utilizadas na execução de cada exame foram aquelas estabelecidas pelo serviço como rotina básica na avaliação do joelho pela RM. As seqüências realizadas utilizaram a técnica spin-eco ponderada em 
DP e T2 no plano sagital, a técnica spineco ponderada em T1 e T2 no plano coronal, a técnica gradiente-eco ponderada em T2* no plano axial e STIR no plano coronal. Em nenhum dos casos estudados administrou-se meio de contraste paramagnético por via endovenosa ou intra-articular.

Em todos os pacientes estudados foram identificados os tipos de lesões ósseas, suas localizações e as maneiras de apresentação nas diferentes seqüências obtidas na RM. Não foi feita, nesta pesquisa, correlação com achados da radiologia convencional, da artroscopia ou da tomografia computadorizada. Avaliou-se, ainda, a frequiência destas lesões, correlacionando-as com o mecanismo de injúria e os dados clínicos. Procurou-se detectar também as lesões de outras estruturas internas do joelho, associadas a essas afecções ósseas. Todos os exames foram revistos por pelo menos dois radiologistas do serviço.

\section{RESULTADOS}

Dos 50 pacientes estudados com história de trauma no joelho, e que foram submetidos ao exame de RM, observouse maior comprometimento dos indivíduos do sexo masculino (84\%) sobre o feminino (16\%). A faixa etária variou de 12 a 77 anos, com média aos 33 anos.

Em nossa casuística, os mecanismos de agressão determinantes das lesões ósseas nos pacientes foram, basicamente, de três tipos: entorse (50\%), trauma direto por forças externas (46\%) e queda da própria altura (4\%).

Os tempos de evolução dos sintomas e da solicitação da RM foram variáveis nos pacientes em estudo, havendo predominância do quadro álgico nos primeiros sete dias após o trauma (24 indivíduos - 48\%). A perpetuação dos sintomas mostrou-se relacionada a processos degenerativos condicionados por instabilidade articular e/ou por subluxações da patela.

$\mathrm{Na}$ dependência das lesões ósseas, ligamentares ou meniscais existentes, os sintomas dos pacientes estudados foram variáveis, sendo encontrados mais de um tipo no mesmo indivíduo. Nas contusões e fraturas ósseas, houve predomínio de pacientes cursando com dor (100\%), associada ou não a limitação ou a dificuldade dos movimentos (34\%), a edema do tecido celular subcutâneo (26\%) e a derrame articular (66\%). Nos casos de condromalácia, foram referidas dor e crepitação à movimentação (4\%).

Os tipos principais de lesões ósseas encontradas foram: a contusão óssea, a fratura osteocondral e a fratura óssea (Tabela 1). A condromalácia foi identificada em indivíduos com história tardia de trauma (cerca de um ano antes), associada a subluxação da patela, determinada pelo trauma anterior. Foram encontradas, de forma conjunta com estas afecções ósseas, as lesões meniscais, ligamentares e tendinosas, o derrame articular e o edema de partes moles.

As contusões ósseas ocorreram em maior número de casos (38 casos $-76 \%$ ). $\mathrm{O}$ envolvimento isolado do fêmur ou da tíbia foi encontrado em 29 pacientes, sendo que em 19 indivíduos foram acometidos os côndilos femorais e em dez casos a lesão óssea se localizava nos platôs tibiais. Também se pôde observar o acometimento do platô tibial e do côndilo femoral de forma conjunta em sete pacientes (Figura 1).

As lesões osteocondrais ocorreram em menor número de casos (cinco casos - 10\%), acometendo apenas os côndilos femorais, sendo quatro localizadas no côndilo femoral medial (Figura 2) e uma no côndilo femoral lateral.

Observaram-se, também, um paciente com fratura plurifragmentar do côndilo medial do fêmur (Figura 3) e outros dois com fratura impactada do côndilo lateral do fêmur, cursando, em um deles, com contusão dos platôs tibiais, e o outro, com contusão do platô tibial lateral.

Dois indivíduos apresentaram contusão óssea da patela, sendo que um deles cursou com subluxação e contusão óssea conjunta do platô tibial medial. Outro paciente mostrou subluxação e arrancamento da cartilagem patelar, com contusão óssea do côndilo femoral lateral e presença de fragmento cartilaginoso livre na bursa suprapatelar. Detectaram-se mais três casos de subluxação da patela, sendo um deles com fratura linear (Figura 4) e os outros dois com condromalácia grau I (Figura 5).

As contusões, as fraturas ósseas e as fraturas osteocondrais foram visibilizadas na RM como áreas de aumento da intensidade de sinal nas seqüências spineco ponderadas em T2, gradiente-eco ponderada em T2* e STIR, e como áreas de baixa intensidade de sinal nas seqüências spin-eco ponderadas em T1 e DP (Tabela 2).

A condromalácia mostrou-se, na RM, como áreas de aumento da intensidade de sinal nas seqüências gradiente-eco ponderadas em $\mathrm{T} 2 *$, e como áreas de baixa

Tabela 1 Tipos de lesões ósseas no trauma do joelho (50 casos).

\begin{tabular}{|l|l|c|}
\hline \multicolumn{1}{|c|}{ Tipos de lesões } & Localização & $\mathrm{N}^{0}$ de casos ( $\left.\mathrm{n}=50\right)$ \\
\hline Contusões & Fêmur & 19 \\
& Tíbia & 10 \\
& Fêmur + tíbia & 7 \\
& Patela & 1 \\
& Tíbia + patela & 1 \\
\hline Fraturas osteocondrais & Fêmur & 5 \\
& Tíbia & - \\
\hline Fraturas & Fêmur & 1 \\
& Tíbia & 1 \\
\hline Fratura + contusão & Patela & 1 \\
& Patela + fêmur & 2 \\
\hline Condromalácia & Fêmur + tíbia & 2 \\
\hline
\end{tabular}




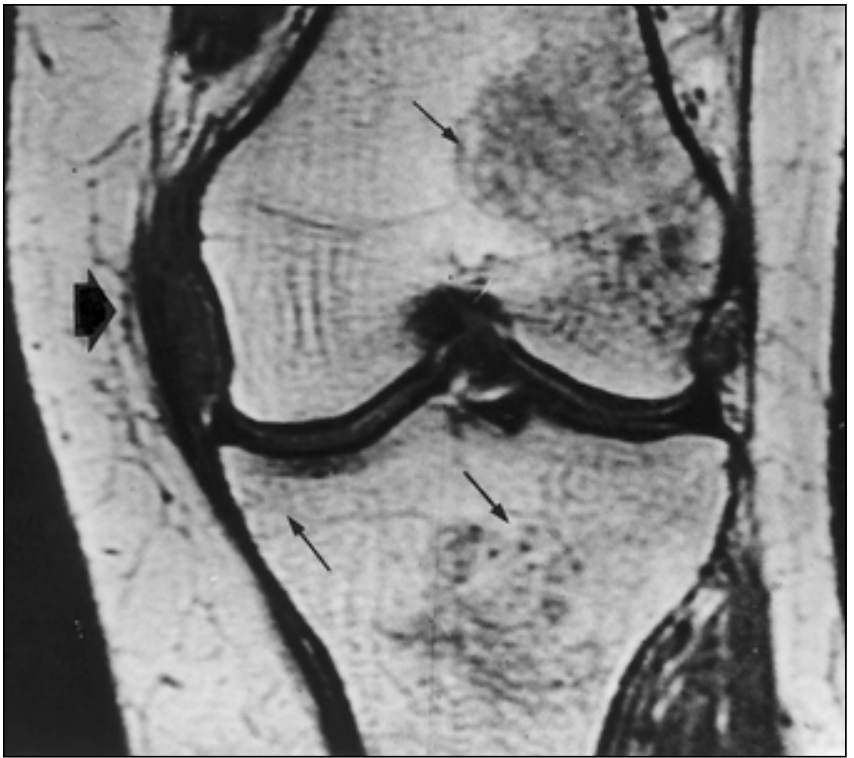

A

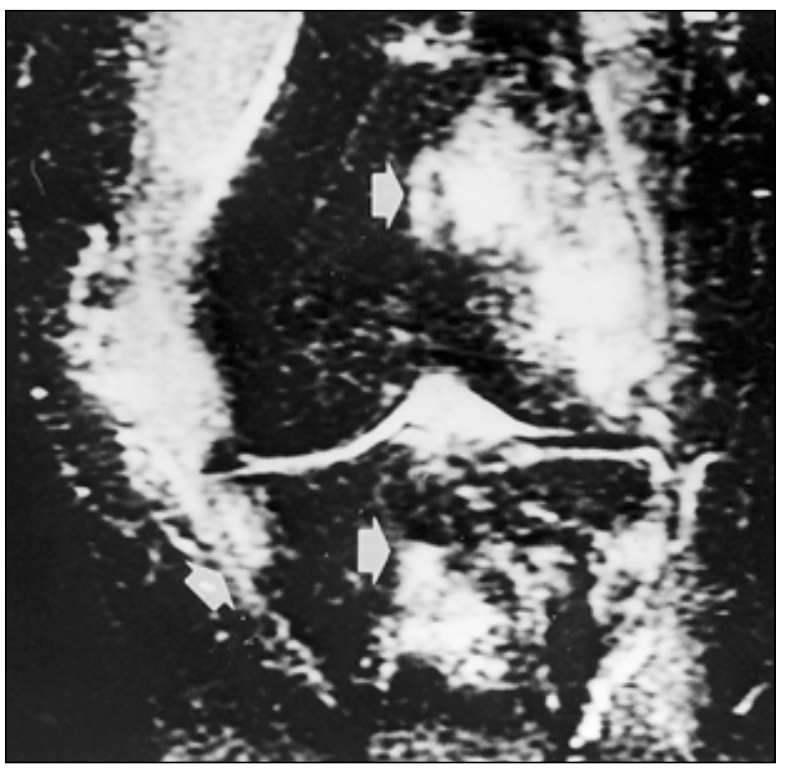

C

Figura 1. Em A, plano coronal ponderado em T1, observam-se contusão do côndilo femoral lateral e platôs tibiais (setas finas) e estiramento do ligamento colateral medial (seta grossa). Em B, mesmo plano ponderado em T2, além dos achados observados em $\mathbf{A}$, nota-se também menisco medial extruso (ponta de seta). Em C, plano coronal com a técnica de supressão de gordura (STIR), observa-se contusão do côndilo femoral e platôs tibiais (setas). Em D, plano sagital ponderado em T2, notam-se estiramento do ligamento cruzado anterior (setas grossas), derrame articular (estrela) e edema do tecido celular subcutâneo anterior ao tendão patelar (seta branca).

Tabela 2 Aspecto das lesões ósseas do trauma do joelho na ressonância magnética.

\begin{tabular}{|l|l|}
\hline \multicolumn{1}{|c|}{ Tipos de lesões } & \multicolumn{1}{|c|}{ Aspectos na ressonância magnética } \\
\hline $\begin{array}{l}\text { Contusões ósseas e fraturas } \\
\text { osteocondrais }\end{array}$ & $\begin{array}{l}\text { Aumento da intensidade de sinal em T2, T2* e STIR; } \\
\text { Baixa intensidade de sinal em T1 e DP }\end{array}$ \\
\hline Fratura & $\begin{array}{l}\text { Aumento da intensidade de sinal em T2, T2* e STIR; } \\
\text { Baixa intensidade de sinal em T1 e DP }\end{array}$ \\
\hline Condromalácia & $\begin{array}{l}\text { Aumento da intensidade de sinal em T2*; } \\
\text { Baixa intensidade de sinal em T1 e DP }\end{array}$ \\
\hline
\end{tabular}

intensidade de sinal nas seqüências spineco ponderadas em T1 e DP, no nível da cartilagem patelar.

\section{DISCUSSÃO}

A faixa etária dos pacientes acometidos pelas lesões ósseas do joelho póstrauma foi extremamente variável nos diversos trabalhos consultados, havendo, 


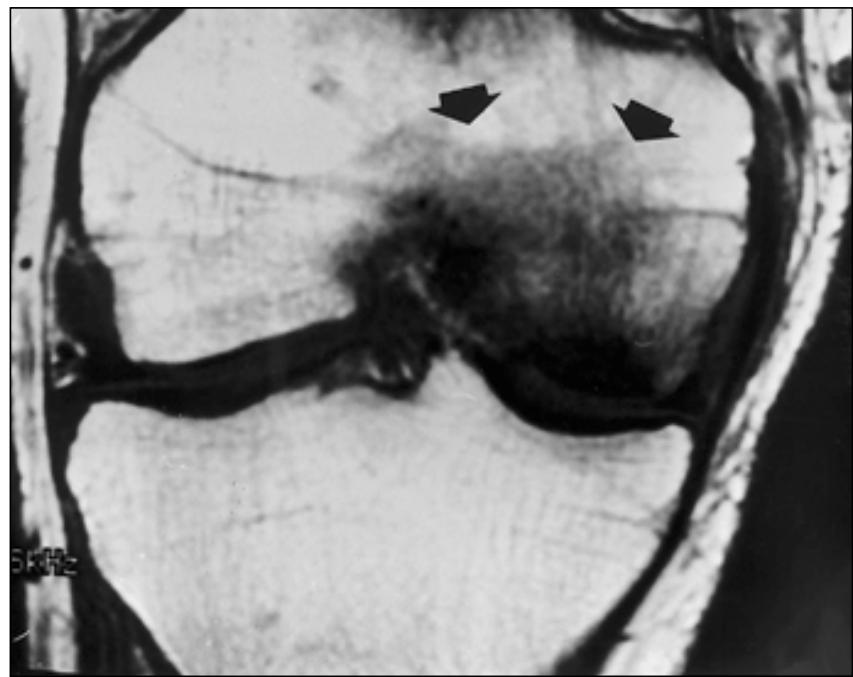

A

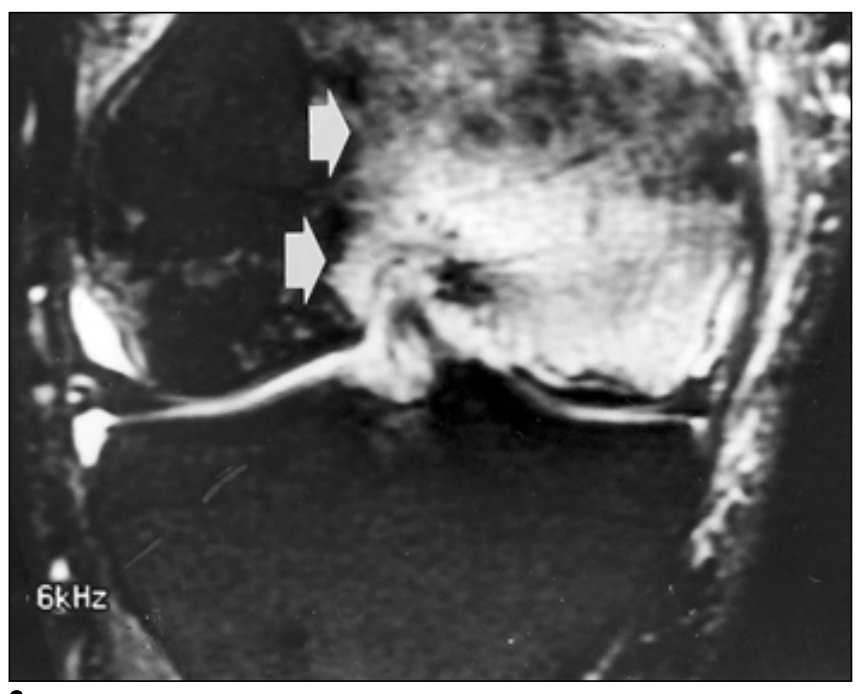

C

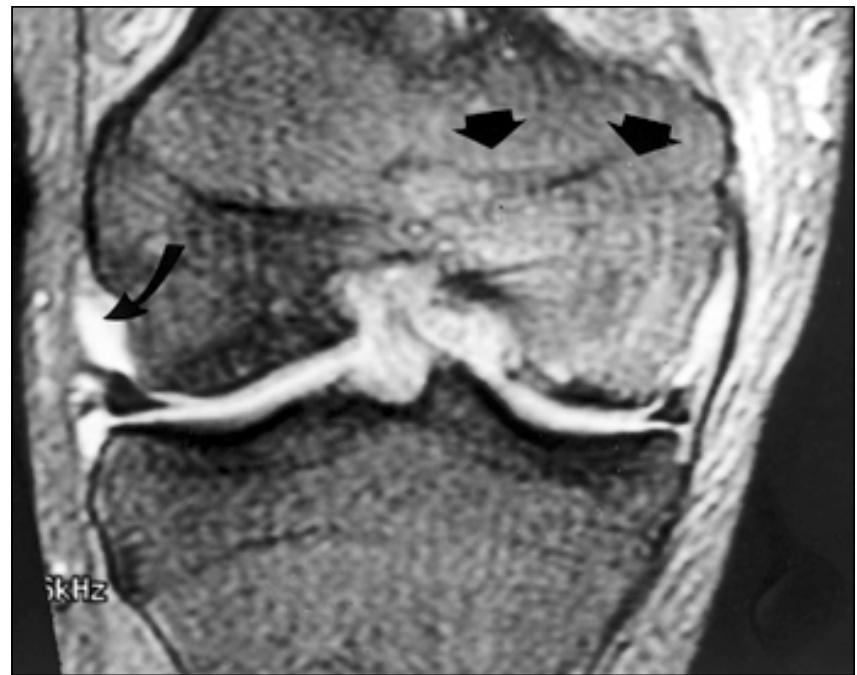

B

Figura 2. Planos coronais ponderados em T1 (A) e em T2 (B) mostram fratura osteocondral no côndilo femoral medial, com extenso edema da medular óssea adjacente (setas). Em B, observa-se também pequeno derrame articular (seta curva). Em C, plano coronal com a técnica de supressão de gordura (STIR), evidencia-se fratura osteocondral no côndilo femoral medial, com extenso edema da medular óssea adjacente (setas). por exemplo, predominância da terceira década de vida no estudo de Lynch et $a l .{ }^{(8)}$, não-concordante com os achados deste estudo, no qual a quarta década de vida foi a mais acometida.

No trabalho de Yao e Lee ${ }^{(9)}$ foram identificados mecanismos de injúria semelhantes aos do nosso estudo. Nos oito pacientes por eles estudados, quatro $(50 \%)$ tiveram trauma direto, dois $(25 \%)$ cursaram com entorse e dois $(25 \%)$ sofreram queda da própria altura. Dos 50 indivíduos por nós analisados, houve predomínio dos casos de entorse, detectados em 25 pacientes $(50 \%), 23(46 \%)$ apresentaram trauma direto e dois (4\%) sofreram queda da própria altura.

O tempo decorrido para a solicitação do exame de RM do joelho após o trau- ma foi variável, havendo predominância nos primeiros sete dias após o acidente (24 casos - 48\%). No estudo de Tervonen et $a l .{ }^{(\mathbf{1 0 )}}$, o tempo médio para a realização deste método de imagem foi de dez dias após o acidente, e para Yao e Lee ${ }^{(9)}$, de 15 dias. Os exames de RM solicitados em pacientes com sintomatologia crônica (cerca de um ano após o trauma) também foram capazes de identificar lesões ósseas cursando com alteração da intensidade de sinal; acreditamos que tal fato seja decorrente da instabilidade articular pós-trauma.

De modo semelhante aos achados de Gartland $^{(11)}$, as contusões e fraturas ósseas dos nossos pacientes cursaram mais comumente com dor (100\%), associada ou não à limitação ou dificuldade dos movimentos, edema do tecido celular subcutâneo (26\%) e derrame articular (66\%). Algumas fraturas ósseas foram acompanhadas também de estalidos no momento do trauma e os casos de condromalácia cursaram com dor e crepitação à movimentação.

Segundo Casteleyn e Handelberg ${ }^{(\mathbf{1 2})}$, cerca de $89 \%$ dos indivíduos com aspectos clássicos de subluxação transitória da patela pós-trauma na RM não foram diagnosticados clinicamente. Semelhante fato também pôde ser notado em nosso estudo, em que dois dos cinco casos que cursaram com subluxação da patela não foram descobertos durante o exame físico, principalmente por causa da presença do edema e da dor intensa durante a mobilização do joelho. 


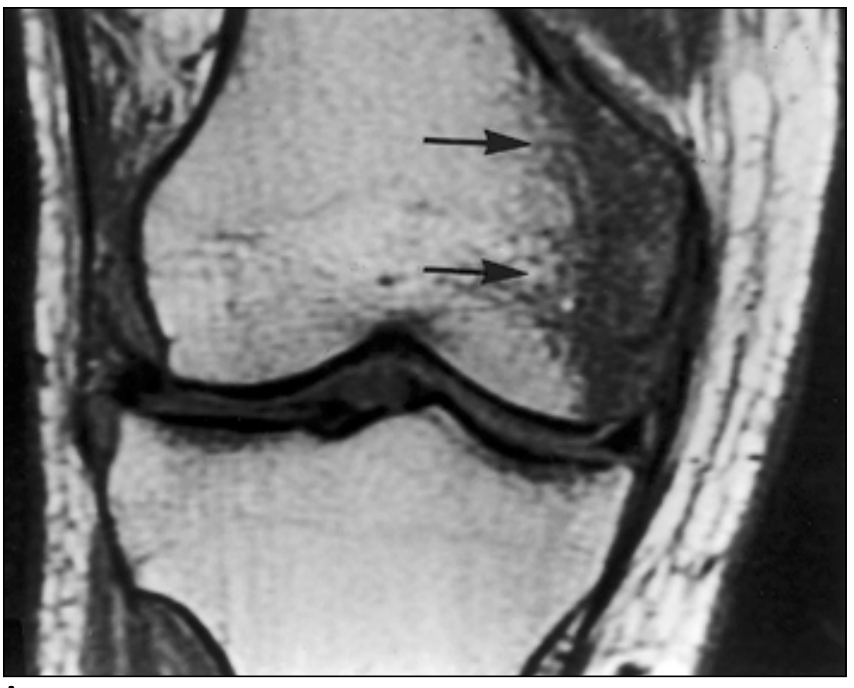

A

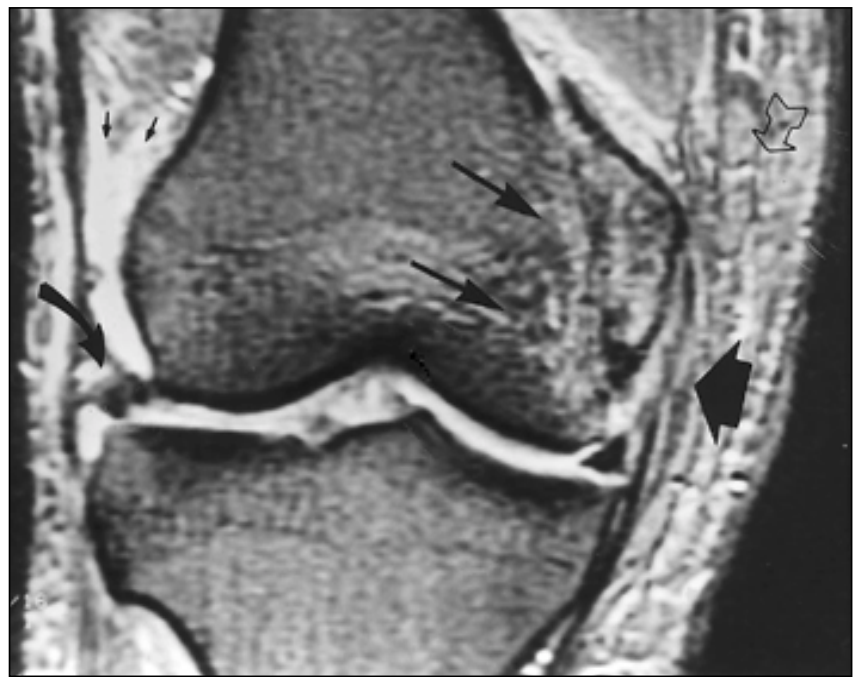

B

Figura 3. Em A, plano coronal ponderado em T1, observa-se fratura plurifragmentar do côndilo femoral medial (setas). Em B, plano coronal ponderado em T2, notam-se fratura plurifragmentar do côndilo femoral medial (setas finas), estiramento do ligamento colateral medial (seta grossa), menisco lateral extruso e macerado (seta curva), edema do tecido celular subcutâneo (contorno de seta) e derrame articular (setas pequenas).

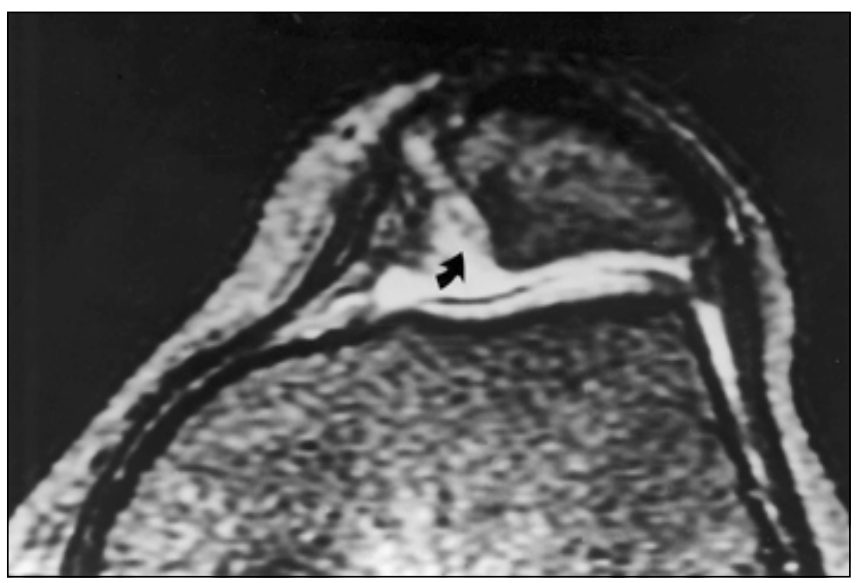

Figura 4. Plano axial ponderado em $\mathrm{T} 2^{*}$ demonstra fratura linear da patela (seta), com discreta subluxação.

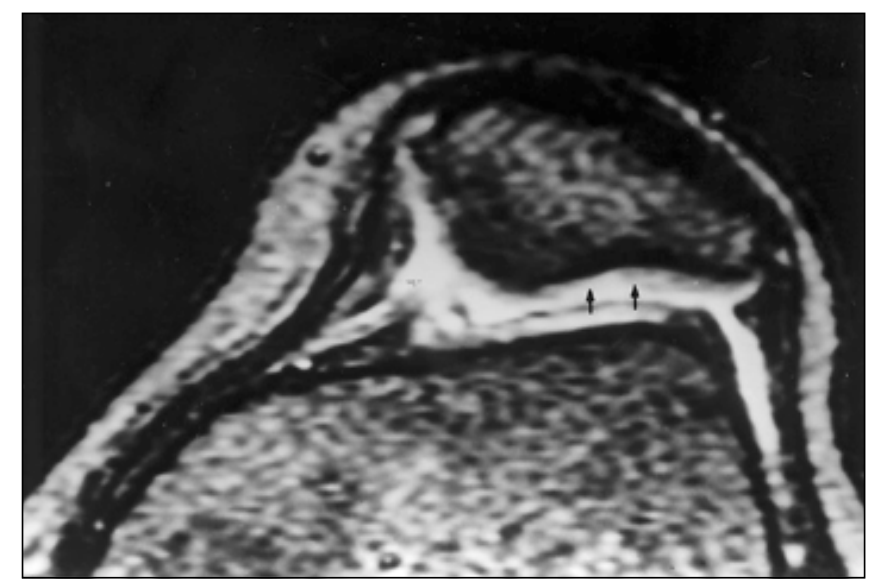

Figura 5. Plano axial ponderado em T2* evidencia condromalácia grau I (setas) e discreta subluxação da patela.
As principais alterações ósseas encontradas nos 50 pacientes estudados com história de trauma agudo do joelho foram as contusões ósseas do fêmur ou da tíbia, de forma isolada ou conjunta (36 indivíduos $-72 \%$ ). Tal fato também foi observado no estudo de Tervonen et al. ${ }^{(\mathbf{1 0})}$, no qual, dos 27 casos descritos, 21 mostraram este mesmo tipo de lesão no côndilo femoral, cinco apresentaram-na na tíbia, e um, na fíbula. No presente estudo, porém, não obtivemos nenhum caso de lesão óssea da fíbula.

De acordo com Vellet ${ }^{(\mathbf{1 3})}$ e Vellet et $a l .{ }^{(14)}$, as fraturas osteocondrais são consideradas a principal causa de sequielas, tendo sido encontradas em $67 \%$ de 21 pacientes estudados com trauma de joelho. Esses mesmos autores referem um maior comprometimento deste tipo de lesão no compartimento lateral do joelho $(81 \%)$. No nosso estudo, as fraturas osteocondrais foram menos freqüentes e ocorreram em cinco pacientes (10\%), acometendo apenas os côndilos femorais, sendo o medial o mais afetado.

As fraturas ósseas foram detectadas em menor número de casos, comprometendo cinco (10\%) dos indivíduos por nós estudados. Os côndilos femorais foram os mais afetados e as fraturas por impactação foram as mais freqüentes.
Apenas três deles apresentaram contusão associada de outros compartimentos ósseos em correspondência. Como a radiografia convencional é capaz de detectar a maioria desses tipos de fratura, a solicitação da RM foi feita para os casos que cursaram com extensão intra-articular e possíveis lesões ligamentares ou meniscais associadas.

Em geral, as contusões e as fraturas da patela ocorrem de forma conjunta ${ }^{(15)}$ e a RM deve ser sempre realizada nestes casos, sendo capaz de demonstrar outras lesões ósseas ou ligamentares envolvidas. Neste estudo, o mecanismo de injúria mais comum foi o trauma direto $(6 \%)$. 
Dos 50 casos estudados, apenas três (6\%) não apresentaram comprometimento ósseo associado a injúrias ligamentares ou meniscais. No estudo de Tervonen et al. ${ }^{(\mathbf{1 0})}$, foram encontrados 17 casos com contusão óssea de forma isolada, correspondendo a $5,6 \%$ dos 302 pacientes estudados.

Algumas das lesões ósseas encontradas não mostraram associação habitual com as lesões ligamentares, meniscais ou mesmo ósseas, normalmente citadas na literatura. Acreditamos que tal fato seja decorrente da ação conjunta de múltiplos mecanismos de injúria.

No nosso estudo, como nos trabalhos de Vellet ${ }^{(13)}$ e Vellet et al. ${ }^{(14)}$, as contusões ósseas e as fraturas osteocondrais ou ósseas, por estarem associadas a edema ou a hemorragia da medular óssea, apresentaram-se como áreas segmentares de aumento de intensidade de sinal nas seqüências spin-eco ponderadas em T2, gradiente-eco ponderadas em $\mathrm{T} 2 *$ e STIR, e como áreas de baixa intensidade de sinal nas sequiências spin-eco ponderadas em T1 e DP.

O tipo de protocolo utilizado para o estudo do joelho na RM ainda é variável em cada instituição, tendo como objetivo obter imagens com a melhor resolução espacial, a menor taxa possível de sinal e ruído, o máximo de contraste e o menor tempo de aquisição. Consideramos satisfatório, para a avaliação dos casos de trauma do joelho, a realização de seqüências multiplanares nos planos axial, coronal e sagital, com imagens ponderadas em T1, T2, DP, T2* e STIR.

Da mesma forma que nos estudos de Stoller ${ }^{(16)}$ e Stoller et al. ${ }^{(17)}$, os casos que cursam com alteração da morfologia do osso foram mais bem avaliados nas seqüências T2* GRE, muitas vezes obscurecidas nas seqüências T1 e STIR, em conseqüência do intenso edema e da hemorragia da medular óssea. As seqüências T2 FSE e T2* GRE mostraram-se úteis na avaliação do líquido intra-articular e das lesões na cartilagem patelar. As seqüências ponderadas em T1 e DP também foram capazes de demonstrar as lesões ósseas, porém de forma menos evidente do que as anteriormente citadas. As sequiências em STIR foram fundamentais para se visibilizar as injúrias ósseas inseridas no interior da gordura, principalmente o edema da medular óssea, pela sua capacidade de supressão da intensidade de sinal normal da gordura.

Neste trabalho, não houve a necessidade da utilização de meio de contraste paramagnético intra-articular diluído, para a avaliação do comprometimento ósseo, sendo que este apresenta indicações limitadas no trauma do joelho, parecendo ser útil nos casos de osteocondrite dissecante pós-traumática, por facilitar o estudo da superfície da cartilagem articular $^{(\mathbf{1}, \mathbf{7}, \mathbf{1 8})}$. Nenhum indivíduo no nosso estudo cursou com esta afecção.

Nos dias de hoje, a RM veio, portanto, redimensionar a avaliação das lesões ósseas do joelho pós-trauma, permitindo o diagnóstico de lesões indetectáveis ou de difícil identificação por outros tipos de exames radiológicos.

\section{REFERÊNCIAS}

1. Coumas JM, Palmer WE. Knee arthrography. Evolution and current status. Radiol Clin North Am 1998;36:703-28.

2. Kaye JJ. Magnetic resonance imaging of the knee. A senior musculoskeletal radiologist's perspective. Magn Reson Imaging Clin N Am 1994;2:497500 .
3. Cundari AM. Estudos do joelho no país do futebol. Jornal da Imagem 1998;(237):14.

4. Firooznia H, Golimbu C, Rafii M. MR imaging of the menisci. Fundamentals of anatomy and pathology. Magn Reson Imaging Clin N Am 1994; 2:325-47.

5. Lotysch M, Mink JH, Schwartz A, Crues JV. Magnetic resonance in the detection of meniscal injuries (Abstract). Magn Reson Imaging 1986;4: 94.

6. Reicher MA, Hartzman S, Duckwiler GR, Bassett LW, Anderson LJ, Gold RH. Meniscal injuries: detection using MR imaging. Radiology 1986; 159:753-7.

7. Vellet AD, Munk PL. Traumatic osteochondral disease of the knee joint and related disorders. In: Munk PL, Helms CA, eds. MRI of the knee. Philadelphia: Lippincott-Raven, 1996:161-88.

8. Lynch TCP, Crues JV, Morgan FW, Sheehan WE, Harter LP, Ryu R. Bone abnormalities of the knee: prevalence and significance at MR imaging. Radiology 1989;171:761-6.

9. Yao L, Lee JK. Occult intraosseous fracture: detection with MR imaging. Radiology 1988; 167:749-51.

10. Tervonen O, Snoep G, Stuart MJ, Ehman RL. Traumatic trabecular lesions observed on MR imaging of the knee. Acta Radiol 1991;32:389-92.

11. Gartland JJ. The knee. In: Gartland JJ, ed. Fundamentals of orthopaedics. Philadelphia: Saunders, 1979:404-31.

12. Casteleyn PP, Handelberg F. Arthroscopy in the diagnosis of occult dislocation of the patella. Acta Orthop Belg 1989;55:381-3.

13. Vellet AD. Magnetic resonance imaging of bone marrow and osteochondral injury. Magn Reson Imaging Clin N Am 1994;2:413-23.

14. Vellet AD, Marks PH, Fowler PJ, Munro TG. Occult posttraumatic osteochondral lesions of the knee: prevalence, classification, and short-term sequelae evaluated with MR imaging. Radiology 1991;178:271-6.

15. Quinn SF, Rose PM, Brown TR, Demlow TA. MR imaging of the patellofemoral compartment. Magn Reson Imaging Clin N Am 1994;2:425-40.

16. Stoller DW. Knee. In: Edelman RR, Zlatkin MB, Hesselink JR, eds. Clinical magnetic resonance imaging. Philadelphia: Saunders, 1996:1954-91.

17. Stoller DW, Cannon WD, Anderson LJ. The knee. In: Stoller DW, ed. Magnetic resonance imaging in orthopaedics \& sports medicine. Philadelphia: Lippincott-Raven, 1997:207-432.

18. Boegard T, Johansson A, Rudling O, Petersson I, Forslind K, Jonsson K. Gadolinium-DTPA-enhanced MR imaging in asymptomatic knees. Acta Radiol 1996;37:877-82. 